DOI: $10.26730 / 1999-4125-2020-6-98-108$

УДК $622+621.22-546$

\author{
РАЗРАБОТКА КРИТЕРИЕВ ОБЕСПЕЧЕНИЯ ГИДРАВЛИЧЕСКИХ \\ ПРОЦЕССОВ В УЗКИХ КАНАЛАХ ГИДРОСИСТЕМЫ ПРИ СОЗДАНИИ \\ НОВЫХ КАРЬЕРНЫХ САМОСВАЛОВ
}

\title{
DEVELOPMENT OF CRITERIA FOR ENSURING HYDRAULIC PROCESSES IN NARROW CHANNELS OF THE HYDRAULIC SYSTEM WHEN CREATING NEW QUARRY DUMP TRUCKS
}

\author{
Панасенков Дмитрий Александрович ${ }^{1}$, \\ магистрант, e-mail: forestbol@bk.ru \\ Panasenkov Dmitry A. ${ }^{1}$, undergraduate, \\ Зайцев Александр Петрович ${ }^{1}$, \\ аспирант, e-mail: zaycev.a.p@ bmstu.ru \\ Zaitsev Alexandr P. ${ }^{1}$, postgraduate, \\ Пикалов Никита Андреевич ${ }^{1}$, \\ аспирант, e-mail: nikitapikalov@bmstu.ru
}

Pikalov Nikita A. ${ }^{1}$, postgraduate,

Карташов Александр Борисович ${ }^{1}$,

канд. техн. наук, e-mail: kartashov@bmstu.ru

Kartashov Alexandr B. ${ }^{1}$, C. Sc. in Engineering,

Дубинкин Дмитрий Михайлович ${ }^{2}$,

канд. техн. наук, доцент, e-mail: ddm.tm@ @uzstu.ru

Dubinkin Dmitry M. ${ }^{2}$, C. Sc. in Engineering, Associate Professor

${ }^{1}$ Московский Государственный Технический Университет им. Н. Э. Баумана, 105005, Россия, г. Москва, 2-я Бауманская улица, д. 5, стр. 1

${ }^{1}$ Bauman Moscow State Technical University, 105005, Russian Federation, Moscow, 2nd

Baumanskaya street, 5

${ }^{2}$ Кузбасский государственный технический университет имени Т.Ф. Горбачева, 650000, Россия, г. Кемерово, ул. Весенняя, 28

${ }^{2}$ T. F. Gorbachev Kuzbass State Technical University, 28, Vesennyaya St., Kemerovo, 650000,

Russian Federation

\section{Аннотация:}

В работе приведен гидродинамический анализ прочессов, возникающих при течении рабочей жидкости в каналах гидравлических блоков гидропривода карьерного автономного автосамосвала на примере блока, обеспечивающего работу гидроаппаратуры тормозной системь. Основное внимание уделяется явлению возникновения кавитационных каверн и гидромеханических потерь в зависимости от параметров рабочей жидкости и геометрических параметров гидравлических каналов. В данной статье показаны модели турбулентности SST-HL и SSG. Также представлена кавитационная модель Рэлея Плессе, встроенная в программный пакет ANSYS CFX.

Ключевые слова: карьерный самосвал, горнопромыциленный транспорт, тяжелая платформа, горные мамины, гидропривод.

Abstract:

The paper presents a hydrodynamic analysis of the processes occurring in the flow of the working fluid in the channels of hydraulic units, hydraulic drive career the Autonomous dump truck, for example unit, providing the hydraulics of the braking system. The main attention is paid to the phenomenon of cavitation cavities and hydromechanical losses depending on the parameters of the working fluid and the geometric parameters of hydraulic 
channels. this article shows the sst-hl and ssg turbulence models. The Rayleigh Plesset cavitation model integrated into the ANSYS CFX software package is also presented.

Key words: mining dump truck, mining transport, heavy platform, mining machine, mining, hydraulic drive.

\section{Введение.}

Развитие

современных карьерных автосамосвалов направлено на улучшение их показателей маневренности, скорости выполнения операций, экологической и эксплуатационной безопасности [1]. Также в настоящее время ведутся активные разработки карьерных автосамосвалов, ориентированные на повышение их уровня автономности $[2,3,4] . \quad$ Конструирование автосамосвалов с шарнирно-сочлененной рамой или с применением поворотных мостов позволяет повысить их маневренность, а применение компонентов с электрическим приводом обеспечивает работу систем в автономном или дистанционном режиме. Немаловажное значение в подобном развитии имеет гидравлическая система автосамосвалов.

Гидросистема современной карьерной техники представляет из себя сложную объединенную структуру, включающую в себя различные источники гидравлической энергии, исполнительные механизмы и средства их активации и управления. Основными исполнительными механизмами в гидравлической системе карьерного самосвала являются гидроцилиндры или гидромоторы рулевого управления, гидроцилиндры подъема грузовой платформы и однополостные камеры рабочей и стояночной тормозной системы. Для обеспечения их нормальной работы необходимо реализовать высокие значения расхода и давления. В то же время применение гидравлических распределителей с электронным управлением позволяет повысить скорость срабатывания гидравлического привода, а также реализовывать автономное иди дистанционное управление его исполнительными механизмами.

При разработке и компоновке гидропривода карьерного автосамосвала часто возникает необходимость обеспечить наиболее компактное и при этом эффективное размещение картриджной клапанной аппаратуры, обеспечивающей работу тормозных механизмов, гидроцилиндров подъема грузовой платформы, гидравлических цилиндров складывания рамы или гидромоторов поворотных мостов. С целью минимизации номенклатуры гидравлических компонентов их объединяют в гидравлические блоки. Основной задачей при разработке таких блоков является обеспечение быстродействия тормозной системы, рулевого управления и подъема грузовой платформы. Эта задача особенно остро стоит при разработке гидросистемы карьерных автосамосвалов, поворот которых обеспечен за счет складывания рамы или поворота мостов, т.к. эффективность и безопасность использования таких транспортных средств при добыче полезных ископаемых в значительной мере зависит от скорости и безотказности функционирования компонентов общей гидросистемы.

В гидросистеме автосамосвалов в настоящее время нашли широкое распространение электромагнитные золотниковые распределители, так как они позволяют реализовывать различные схемы управления $[5,6]$. Помимо процесса трения, приводящего к постепенному разрушению подвижных частей золотникового распределителя [7], существует неизбежный эрозионный износ, возникающий вследствие попадания в золотник частиц металла. Одной из причин попадания частиц металла в рабочую жидкости гидропривода является кавитационная эрозия [8]. В процессе кавитации возникает нарушение сплошности потока жидкости в зоне пониженного давления, сопровождающееся местными гидравлическими ударами, которые возникают при исчезновении кавитационных каверн. Длительное воздействие такой эрозии приводит к постепенному разрушению стенок каналов и отрыву мелких частиц металла, которые попадают в золотниковые распределители гидропривода, что оказывает эрозионное воздействие на компоненты золотника. В работе [9] показано, что эффект износа в гидравлических клапанах приводит к ухудшению работы золотникового распределителя, уменьшает срок его службы, приводит к утечкам в системе.

Поскольку эрозионное воздействие на золотник является следствием кавитации в гидравлических каналах, то при разработке гидроблоков особое внимание следует уделять снижению вероятности кавитационнных явлений. Так, в работе [10] демонстрируется расчетная модель для оценки кавитационно-эрозионных повреждений в соленоидных клапанах дизельного инжектора. В работе [11] авторам удалось снизить погрешность расчета при моделировании процесса кавитации в дроссельном канале за счет приближения расчетной модели к реальным условиям работы гидропривода.

Необходимо отметить, что процесс выбора рациональных параметров каналов гидроблоков осложняется большим их количеством; так, явление кавитации зависит от геометрических параметров каналов, материала корпуса гидравлического блока, давления рабочей жидкости, типа рабочей жидкости и качества внутренних поверхностей каналов (шероховатости).

Испытания, проводимые в работах [12, 13, 14], показали, что выбор материала изделий оказывает 


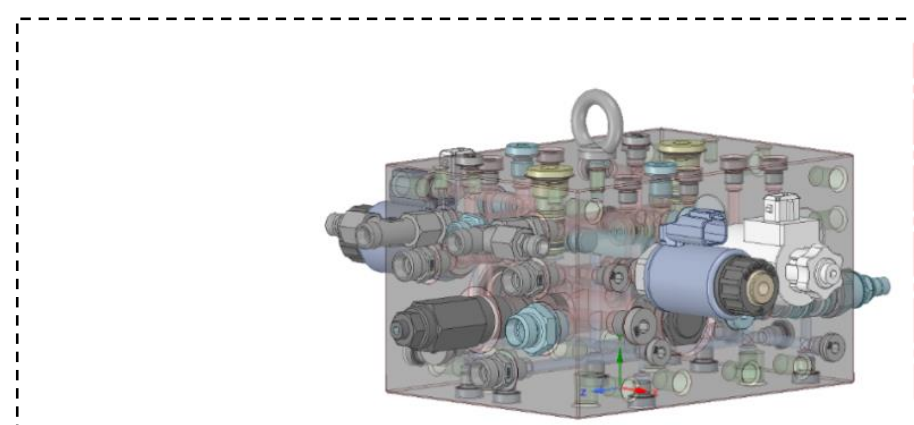

a)

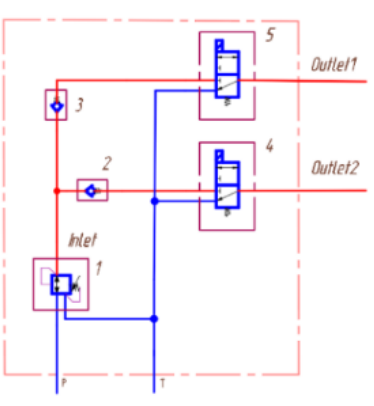

б)

Рис. 1. Гидравлический блок карьерного автосамосвала:

a-геометрическая модель; б - гидравлическая схема исследуемого участка гидравлического блока

Fig. 1. Hydraulic block of a dump truck:

$a$-geometric model; $\sigma$ - hydraulic diagram of the studied section of the hydraulic block

существенное воздействие на процесс

возникновения кавитации, а в работе [15] показано, что этап компьютерного моделирования процесса кавитации необходим для исключения метода проб и ошибок во время проектирования и сокращает время общего процесса разработок.

Одним из критериев эффективной работы гидропривода является его быстродействие, что тесно связано с такими характеристиками рабочего потока жидкости, как скорость потока и его расход. В работе $[16,17,18,19]$ приведены исследования, показывающие зависимость между параметрами потока жидкости: расходом, перепадом давления, скоростью и параметрами самой рабочей жидкости: температурой и вязкостью. Анализ и расчет геометрии гидравлических каналов с учетом параметров рабочей жидкости позволяет выбрать необходимое значение диаметров рабочих каналов так, чтобы скорость срабатывания исполнительных механизмов была максимальной.

\section{Анализ параметров рабочей жидкости и возникновения кавитации в каналах гидравлического блока}

Как отмечалось ранее, при проектировании гидравлических блоков гидропривода карьерного автосамосвала необходимо учитывать значительное количество факторов, влияющих на работу исполнительных механизмов. В работе проводится анализ геометрических параметров гидравлических каналов блоков на примере гидроблока тормозной системы, обеспечивающего размещение и совместную работу клапанной аппаратуры тормозного гидропривода карьерного автосамосвала. Гидравлический привод тормозной системы обладает определенными требованиями к эффективности и скорости работы, характерными для карьерного автосамосвального транспорта. В отличие от гидропривода системы рулевого управления или системы подъема грузовой платформы рассматриваемый гидропривод тормозной системы имеет наименьшие проходные сечения рабочих каналов ввиду меньшего требуемого расхода и высокой скорости течения рабочей жидкости.

На рис. 1 a изображена геометрическая модель разработанного гидравлического блока для карьерного автосамосвала полной массой 50 тонн. Определение параметров каналов данного блока проводилось для прогнозирования характера течения жидкости при разных значениях вязкости, которые, в свою очередь, зависят от температуры. Также были получены значения давления на стенки каналов блока, которые могут быть использованы в прочностных расчетах для рационального выбора материала корпуса гидравлического блока и, как следствие, уменьшить значение деформации для сокращения объема утечек. Кроме того, рассмотрено влияние шероховатости при разных значениях вязкости на выходные параметры (гидромеханические потери). Последнее поможет выбрать оптимальное качество поверхности каналов блока, чтобы найти баланс между стоимостью, КПД и влиянием на вероятность появления кавитации.

На рис. 16 изображена схема участка гидравлического блока, для которого проведено расчетное исследование. Красным цветом показаны те каналы, которые будут задействованы в модели при условии, что клапаны 2, 3, 4, 5 нормально открыты, а через клапан 1 поступает жидкость с постоянным расходом и давлением 70 бар. Помимо расчета сопротивлений и изучения влияния вязкости на характер течения рабочей жидкости в каналах в работе исследован опасный участок канала гидроблока с целью прогнозирования возникновения кавитации.

\section{Описание теоретических предпосылок}

Для определения характера течения жидкости в каналах гидравлического блока на этапе его проектирования и для предотвращения нежелательных явлений, таких как кавитация, а также для учета гидромеханических потерь был проведен расчетный гидродинамический анализ (CFD анализ) в программном комплексе Ansys CFX 19.2.

В первом приближении в качестве расчетной 


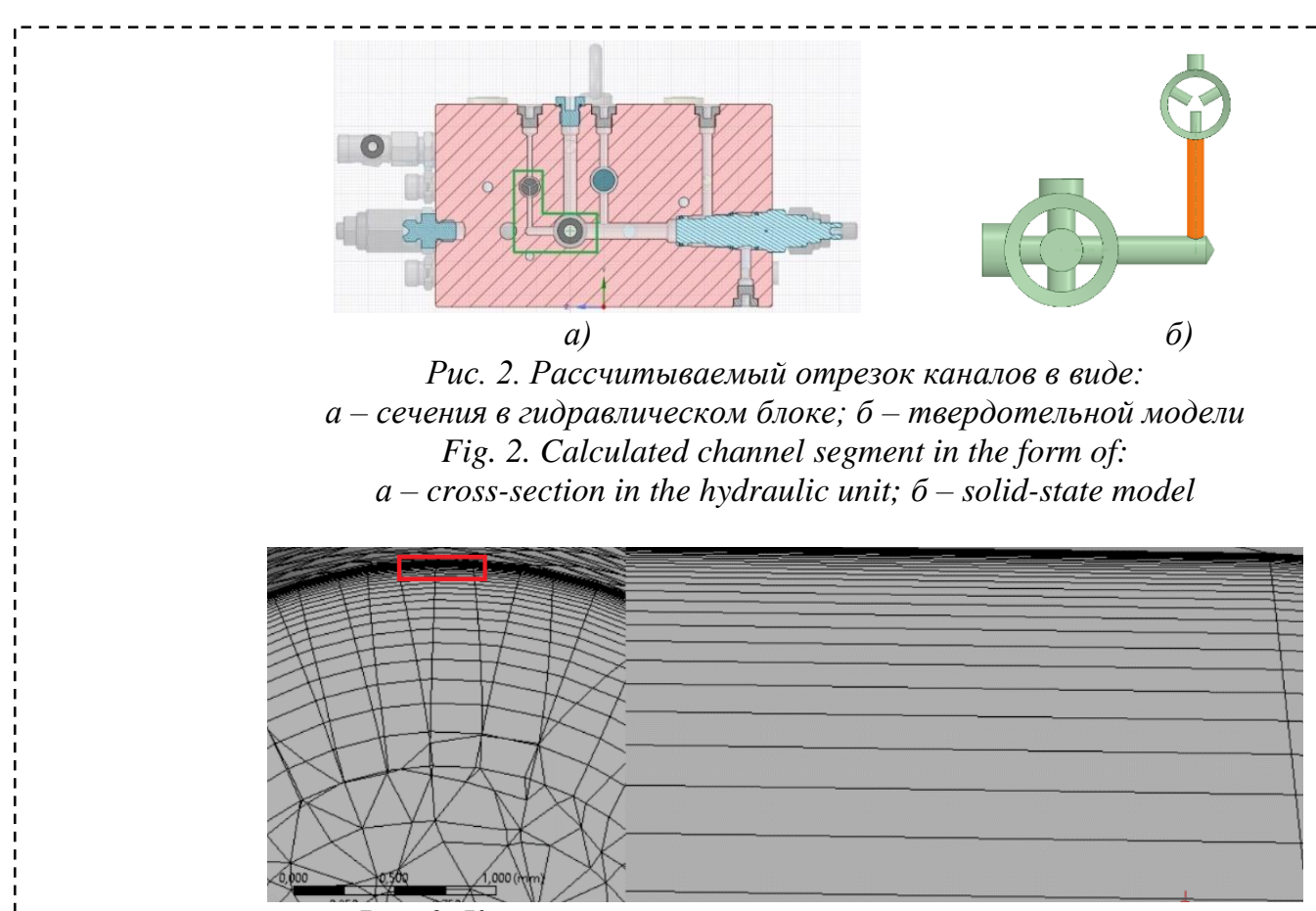

Рис. 3. Конечно-элементная сетка при расчете кавитации

Fig. 3. Finite element grid for cavitation calculation

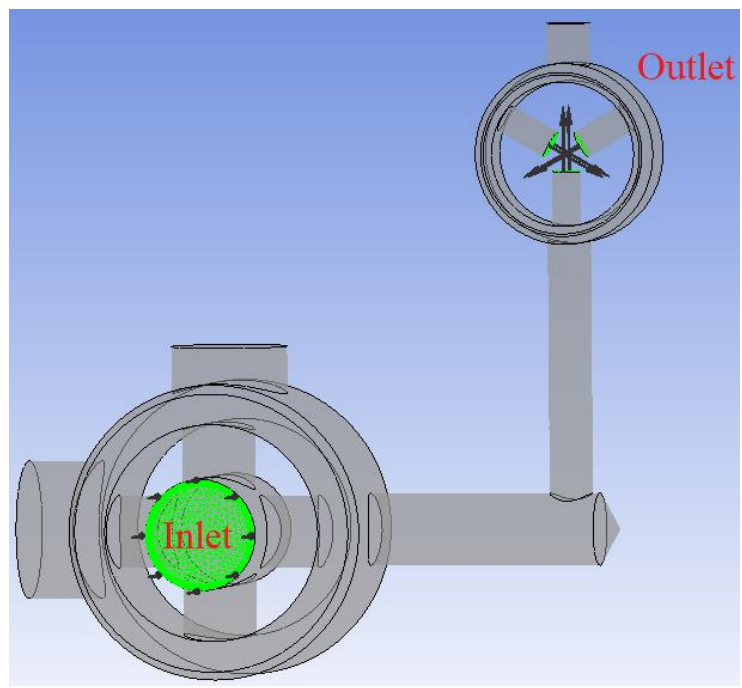

Рис. 4. Модель для расчета кавитачии

Fig. 4. Model for calculating cavitation

модели турбулентности была выбрана модель SSTHL (Shear Stress Transport), в которой используются одновременно две модели турбулентности типа $\mathrm{k}-\varepsilon$ и типа kмаксимально достоверно ведет себя в пограничном слое, а модель турбулентности типа $\mathrm{k}-\varepsilon$ наилучшим образом проявляет себя в ситуации перехода течения жидкости к свободному потоку. Модификация HL, являющаяся дополнением в модели турбулентности SST, позволяет наиболее точно провести моделирование отрыва потока жидкости от стенки канала.

Во втором приближении в качестве расчетной была использована модель напряжений
Рейнольдса, а именно модель SSG (Reynoldsstress). Данная модель используется для расчетов более сложных инженерных задач, поскольку модели типа $\mathrm{k}-\varepsilon$ и типа $\mathrm{k}-\omega$ имеют существенные недостатки в реальных турбулентных потоках. Одной из наиболее важных функций в модели напряжений Рейнольдса является корреляция давление - деформация, $\Phi_{i j}$. Она используется, чтобы стимулировать турбулентность к изотропному состоянию, перераспределяя напряжения Рейнольдса.

Член деформации давления можно разделить на две части:

$$
\Phi_{i j}=\Phi_{i j, 1}+\Phi_{i j, 2}
$$




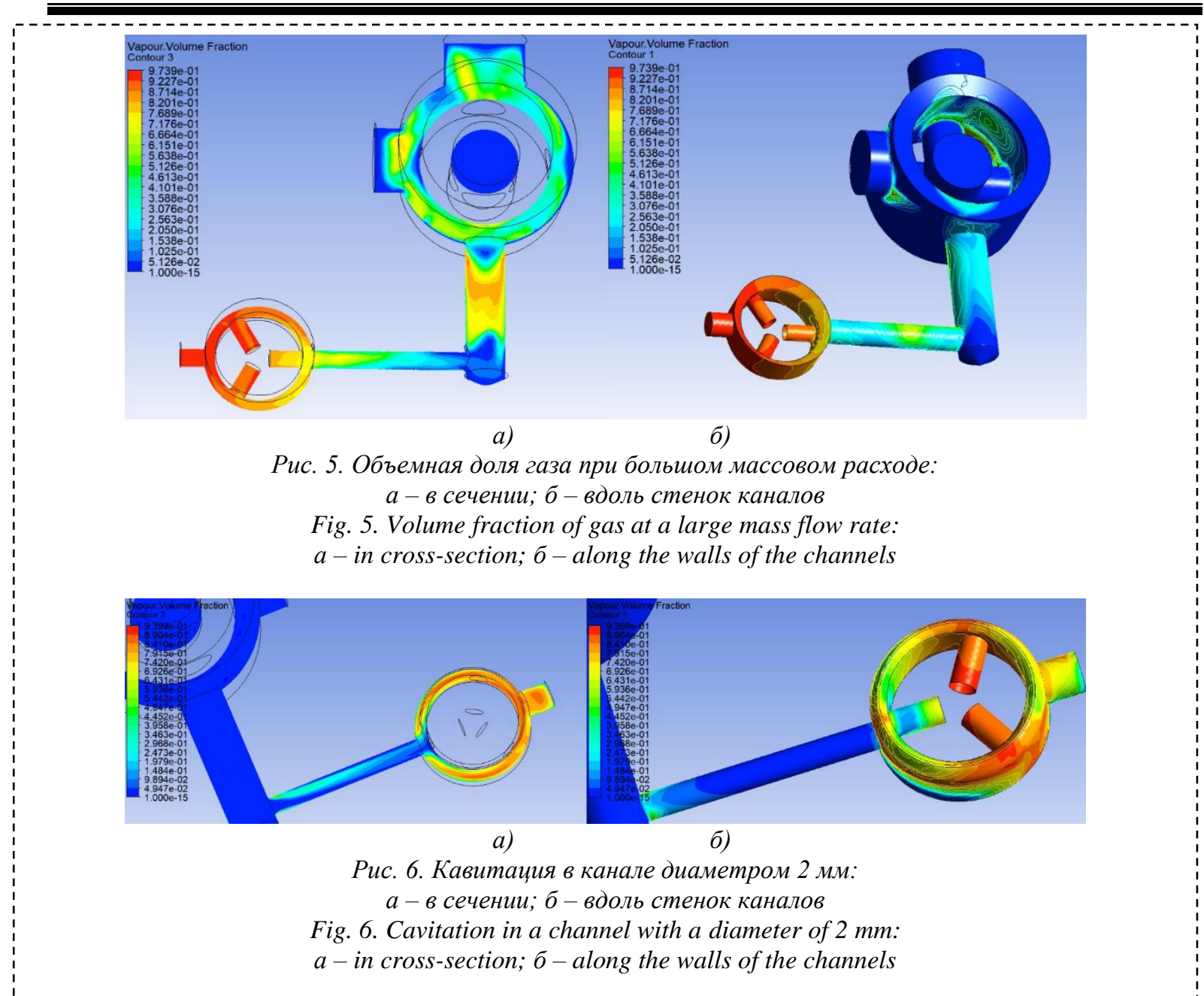

где $\Phi_{i j, 1}-$ «медленная» составляющая, также известная как термин «возврат к изотропии»; $\Phi_{i j, 2^{-}}$ «быстрая» составляющая уравнения.

В модели SSG, в отличии от других моделей Рейнольдсовых напряжений, в которых корреляция давления-деформации является линейной, используются квадратичные соотношения корреляции давления-деформации:

$$
\begin{aligned}
\Phi_{i j, 1}=-\rho \varepsilon\left[C_{s 1} a_{i j}\right. & \\
& +C_{s 2}\left(a_{i k} a_{k j}\right. \\
& \left.\left.-\frac{1}{3} a_{m n} a_{m n} \delta_{i j}\right)\right] \\
\Phi_{i j, 2}=-C_{r 1} P a_{i j} & +C_{r 2} \rho k S_{i j} \\
& -C_{r 3} \rho k S_{i j} \sqrt{a_{m n} a_{m n}} \\
& +C_{r 4} \rho k\left(a_{i k} S_{j k}\right. \\
& +a_{j k} S_{i k} \\
& \left.-\frac{2}{3} a_{k i} S_{k i} \delta_{i j}\right) \\
& +C_{r 5} \rho k\left(a_{i k} \Omega_{j k}\right. \\
& \left.+a_{j k} \Omega_{i k}\right)
\end{aligned}
$$

где $\rho$ - плотность, $\Omega_{i j}-$ тензор завихренности, $a_{i j}$ - тензор анизотропии, $\Phi_{i j}$ - корреляция между давлением и деформацией, $\overline{u_{\imath} u_{\jmath}}-$ тензор рейнольдсовых нап ряжений, $\varepsilon$ - скорость диссипации кинетической энергии турбулентности, $\rho-$ плотность жидкости, $C_{r 1}, C_{r 2}, C_{r 3}, C_{r 4}, C_{r 5}, C_{s 1}, C_{s 2}$ - константы, $S_{i j}-$ средняя скорость деформации, $\delta_{i j}-$ коэффициент Кронекера, $k$ - средняя турбулентная кинетическая энергия, $U_{i}, U_{j}$ - скорости.

В расчетах гидравлического блока, в каналах которого возможно возникновение кавитации, помимо модели турбулентности также использовалась модель кавитации. При вычислении кавитации предварительно проводился расчет для канала с полностью сходящимся решением без модели кавитации. В AnsysCFX в качестве кавитационной модели используется модель Рэлея-Плессе, реализованная в многофазной структуре как модель межфазного массопереноса, которая учитывалась при расчетах, описанных в пунктах 2.2 и 2.3 .

\section{Описание модели при моделировании} кавитации

Первоначально в исследовании был выполнен расчет каналов гидравлического блока, изображенного на рис. 2, на предмет возникновения кавитации при разных значениях массового расхода и различной геометрической формы канала (рис. 2, б), а именно влияние диаметрального размера узкого канала и разных значений шероховатости. 


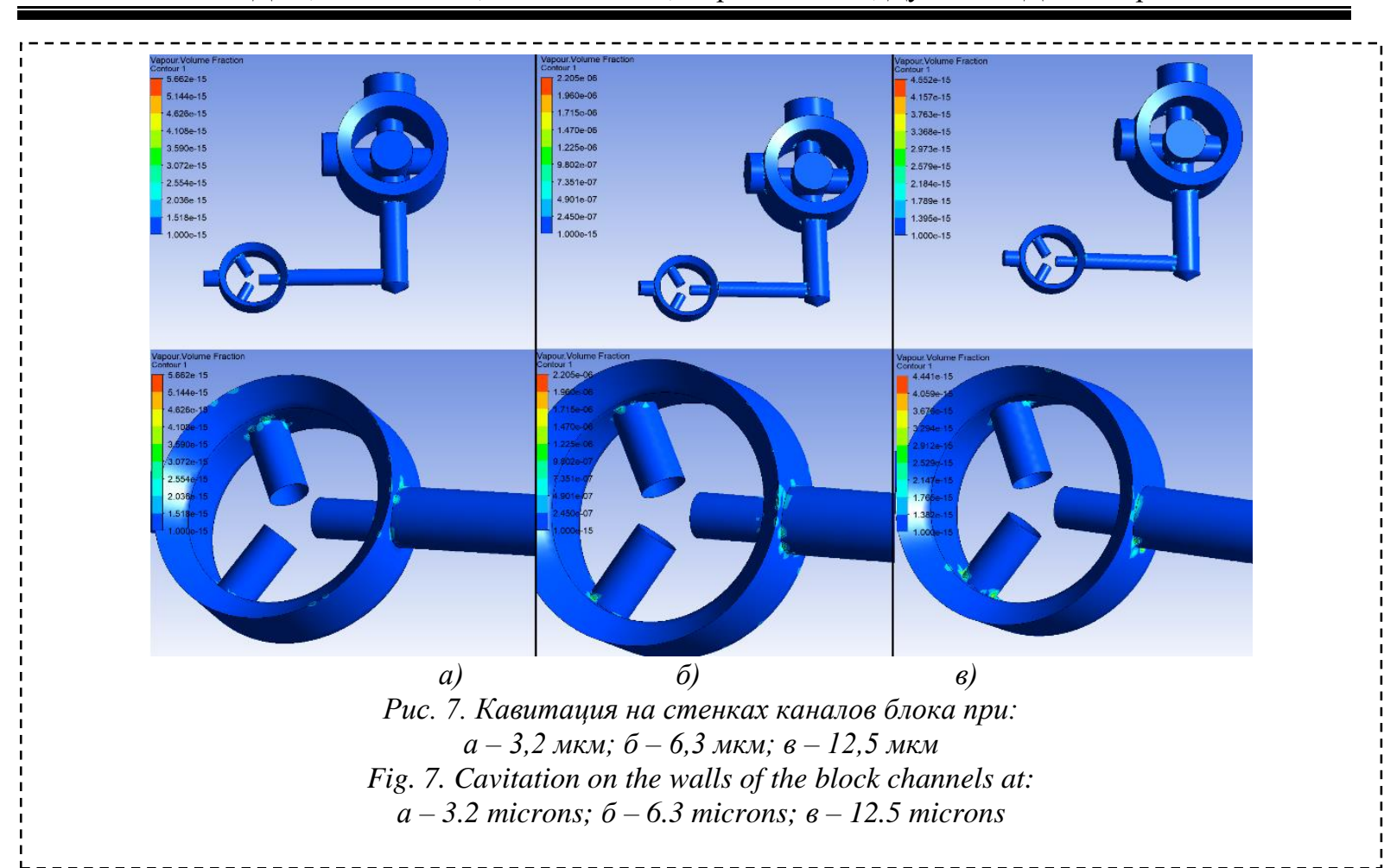

При построении конечно-элементной сетки (рис. 3) в программном комплексе Ansys CFX была использована функция Inflation вдоль стенок каналов для более точного определения режима течения жидкости в пограничном слое и получении более точных решений при использовании выбранных моделей турбулентности SST-HL и SSG и модели кавитации Рэлея-Плессе.

Один из способов задания граничных условий с наибольшей вероятностью сходимости - это задание давления жидкости на входе и ее расхода на выходе. В качестве граничных условий на входе Inlet было задано давление «TotalPressure», а на выходе Outlet был задан массовый расход «MassFlowRate» (рис. 4). Входное отверстие в данной модели является внутренним каналом обратного клапана 3, изображенного на рисунке 2, а выходными отверстиями является вход в каналы клапана 5. Аналогичный расчет для клапанов 3 и 4 не требуется, поскольку каналы являются абсолютно идентичными по форме и габаритам, и расположены симметрично.

\section{Оценка процесса возникновения кавитации в гидравлических каналах}

Первичный расчетный анализ участка каналов распределительного блока, изображенного на рисунке 5, был произведен при температуре рабочей жидкости $100^{\circ} \mathrm{C}$ (динамическая вязкость,


расходах на выходных сечениях в 1 кг/с, давлении на входе 70 бар, величины шероховатости стенок 6,3 мкм, значении давления насыщенного пара 1250 Па и диаметром подводящего канала, выделенного оранжевым цветом на рисунке 2 б, равного 3 мм. Объемная доля газа при вышеперечисленных условиях изображена на рисунке 5.

После выбора клапанов, которые ограничивают максимальный расход (см. рис. 1 б, клапаны 4 и 5), был произведен дополнительный расчет с целью определения возникновения кавитаций в подводящих к клапанам каналах с первоначальным диаметром равным 2 мм (см. рис.2, б). Расчет проводился при аналогичных условиях, за исключением расхода на выходе, который теперь был составлял 30 л/мин. В результате расчета получено, что значение объемной доли газа в подводящем канале свидетельствует о наличии в нем кавитации (рис. $6)$.

В дальнейшей разработке с целью снижения кавитационных явлений было принято решение увеличить диаметр подводящих к клапанам каналов до 3 мм. Далее приведены результаты расчетов кавитации в выбранных каналах при аналогичных граничных условиях и значениях шероховатости поверхности каналов 3,2, 6,3 и 12,5 мкм (рис. 7).

По результатам проведенного расчетного моделирования окончательно были утверждены следующие параметры гидроблока: диаметры подводящих каналов - 3 мм; ограничение расхода в клапанах - 30 л/мин. При утвержденных параметрах объемная доля газа даже при температуре $100^{\circ} \mathrm{C}$, которая для рассматриваемого гидропривода не является рабочей, весьма несущественна и не способна привести к сильным разрушениям гидроблока. Дальнейшие расчеты были произведены при утвержденных параметрах. 


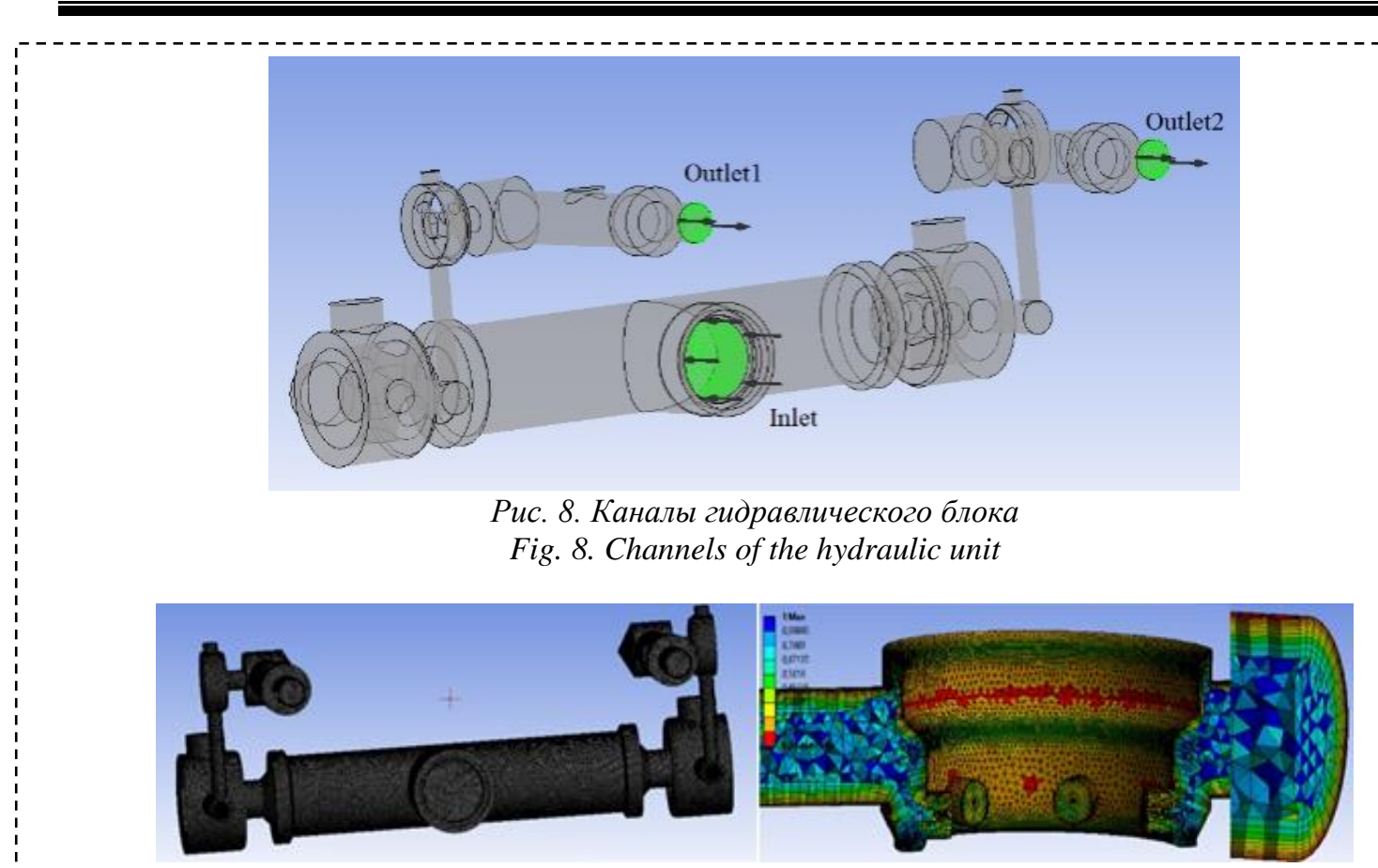

Рис. 9. Конечно-элементная сетка каналов

Fig. 9. Finite element channel grid

Таблица 1. Эмпирическая зависимость динамической вязкости и плотности рабочей жидкости от температуры при давлении 70 бар

Table 1. Empirical dependence of the dynamic viscosity and density of the working fluid on the temperature at a pressure of $70 \mathrm{bar}$

\begin{tabular}{|l|c|c|c|c|}
\hline Среднее значение температуры жидкости, ${ }^{\circ} \mathrm{C}$ & Минус 10 & 30 & 60 & 100 \\
\hline Динамическая вязкость, Па·с & 0,62 & 0,06 & 0,02 & 0,007 \\
\hline Плотность, кг/м ${ }^{3}$ & 894 & 864 & 840 & 810 \\
\hline
\end{tabular}

Описание расчетной модели при исследовании влияния температуры жидкости и шероховатости каналов гидравлического блока на выходные параметры

На рис. 8 изображена модель каналов исследованного участка гидравлического блока (см. рис. 1 б). Каналы представляют из себя цилиндрические отверстия, выполненные в различных направлениях так, чтобы рабочая жидкость, войдя во входной порт, вышла из выходных портов с новыми рабочими характеристиками, последовательно пройдя через различные гидравлические распределители и клапаны.

При построении конечно-элементной сетки (рис. 9) так же, как и при расчете кавитационных режимов, была использована функция Inflation, т. к. расчет проводился с использованием моделей турбулентности SST-HL и SSG.

В качестве граничных условий на входе было задано давление «TotalPressure», на выходах Outlet1 и Outlet2 (см. рис. 2), был задан массовый расход «MassFlowRate». Расход на выходах был установлен одинаковым, поскольку клапаны, которые в нашей модели учтены как полностью открытые, должны открываться синхронно друг с другом, имея при этом одинаковые сопротивления на выходе из гидравлического блока. Для определения гидромеханический потерь в каналах гидравлического блока в настройках расчетной модели задавалось значение шероховатости стенок каналов. Расчет проводился в стационарной постановке, процесс адиабатический.

\section{Анализ результатов расчета}

Расчет был произведен для рабочих каналов гидравлического блока (см. рис. 2) с шероховатостью стенок каналов 3,2, 6,3 и 12,5 мкм, при температурах минус 10, плюс 30,60 и $100^{\circ} \mathrm{C}$ при заданном давлении на входе 70 бар и расходах на выходах 30 л/мин. Максимальная температура, равная $100^{\circ} \mathrm{C}$, была учтена при расчете несмотря на то, что данный температурный режим не является рабочим для гидравлической системы карьерного автосамосвала. Данный температурный режим рассматривается для оценки кавитационного запаса в рассматриваемом гидравлическом блоке, что обеспечит надежность работы гидропривода в случае кратковременного выхода из строя теплообменника системы. Значения вязкости и плотности рабочей жидкости при разных температурах были установлены эмпирическим путем и представлены в табл. 1 .

Исходя из вышеперечисленных условий, 
Вестник Кузбасского государственного технического университета. 2020. № 6, с.98-108

Панасенков Д.А., Зайцев А.П., Пикалов Н.А., Карташов А.Б., Дубинкин Д.М. Разработка ...

Таблица 2. Значения скорости и давления потока рабочей жидкости

Table 2. Working fluid flow velocity and pressure values

\begin{tabular}{|c|c|c|c|c|c|c|c|}
\hline \multirow{2}{*}{$\begin{array}{c}\text { Температура } \\
\text { рабочей } \\
\text { жидкости, }{ }^{\circ} \mathrm{C}\end{array}$} & \multirow{2}{*}{$\begin{array}{c}\text { Шероховатость, } \\
\text { мкм }\end{array}$} & \multicolumn{3}{|c|}{ Скорость, м/с } & \multicolumn{3}{|c|}{ Давление, бар } \\
\hline & & Inlet & Outlet1 & Outlet2 & Inlet & Outlet1 & Outlet2 \\
\hline \multirow{3}{*}{-10} & 3,2 & 4,92 & 10,28 & 10,29 & 69,87 & 43,20 & 43,29 \\
\hline & 6,3 & 4,92 & 10,28 & 10,29 & 69,87 & 43,19 & 43,29 \\
\hline & 12,5 & 4,92 & 10,28 & 10,29 & 69,87 & 43,17 & 43,27 \\
\hline \multirow{3}{*}{30} & 3,2 & 4,84 & 10,31 & 10,31 & 69,89 & 48,92 & 48,46 \\
\hline & 6,3 & 4,84 & 10,31 & 10,31 & 69,89 & 48,90 & 48,44 \\
\hline & 12,5 & 4,84 & 10,31 & 10,31 & 69,89 & 48,86 & 48,40 \\
\hline \multirow{3}{*}{60} & 3,2 & 4,82 & 10,49 & 10,42 & 69,89 & 48,66 & 48,55 \\
\hline & 6,3 & 4,82 & 10,49 & 10,42 & 69,89 & 48,61 & 48,50 \\
\hline & 12,5 & 4,82 & 10,50 & 10,43 & 69,89 & 48,51 & 48,38 \\
\hline \multirow{3}{*}{100} & 3,2 & 4,81 & 10,70 & 10,57 & 69,89 & 48,68 & 48,67 \\
\hline & 6,3 & 4,81 & 10,60 & 10,55 & 69,89 & 48,39 & 48,63 \\
\hline & 12,5 & 4,81 & 10,63 & 10,69 & 69,89 & 48,35 & 48,19 \\
\hline
\end{tabular}

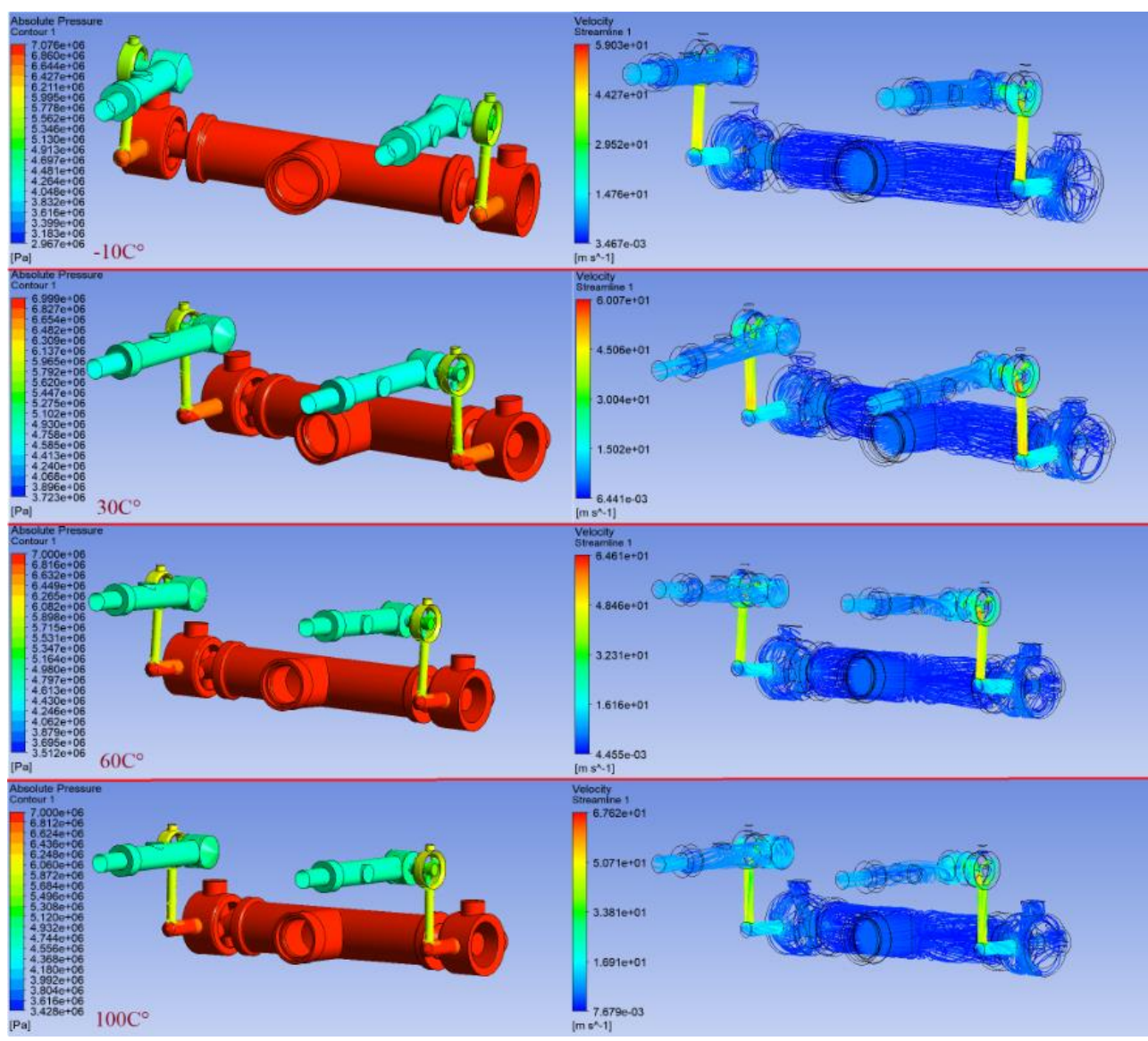

a)

б)

Рис. 10. Характеристика:

$a$-распределения давления на стенки гидравлического блока; б - линии тока в каналах гидравлического блока с отображением значения скорости течения жидкости

Fig. 10.. Characteristic:

$a$-pressure distribution on the walls of the hydraulic unit; $b$-current lines in the channels of the hydraulic unit with the display of the value of the fluid flow rate

были получены значения скорости и давления вязкости жидкости и шероховатости стенки. рабочей жидкости, осредненные по площади входного и выходных сечений гидравлического блока в зависимости от температуры, то есть Данные представлены в табл. 2.

По полученным результатам можно оценить, какое оказывает влияние определенное значение 
шероховатости на выходные параметры при соответствующем значении температуры и динамической вязкости.

На рис. 10 изображены линии тока с распределением скорости жидкости по каналам и давление вдоль стенки для выбранной шероховатости при разных температурных режимах и значении шероховатости 6,3 мкм.

При проектировании гидравлического блока, опираясь на данные, приведенные в таблице 2 и на рисунке 7, была выбрана величина шероховатости стенок канала, равная 6,3 мкм.

\section{Заключение}

В результате проведенного расчетного гидродинамического анализа разрабатываемого распределительного гидравлического блока карьерного автономного автосамосвала была выявлена интенсивность появления кавитационных каверн при различных параметрах, таких как шероховатость поверхности, температурный режим, расход жидкости и диаметр подводящего канала. Также установлена зависимость потерь от величин шероховатости и температуры (вязкости). Благодаря проведенным расчетам удалось потенциально существенно уменьшить кавитацию при температуре рабочей жидкости $100^{\circ} \mathrm{C}$, тем самым обеспечить большой кавитационный запас в гидросистеме автосамосвала, избежать явление суперкавитации и исключить ее при других температурных режимах, а также выбрать рациональные геометрические параметры каналов гидравлического блока и рабочую жидкость гидросистемы карьерного автосамосвала.

Работа выполнена при финансовой поддержке Министерства науки и выстего образования Российской Федерации в рамках соглашения № 075-11-20 19-034 от 22.11.2019г. с ПАО «КАМАЗ» по комплексному проекту «Разработка и создание высокотехнологичного производства автономных тяжельх платформ для безлюдной добычи полезных ископаемых в системе «Умный карьер», при участии ФГБОУ ВО «Кузбасский государственный технический университет имени Т. Ф. Горбачева» в части выполнения научно-исследовательских, опьтноконструкторских и технологических работ.

\section{СПИСОК ЛИТЕРАТУРЫ}

1. А. А. Кулешов, Проектирование и эксплуатация карьерного автотранспорта. Справочник. Ч. 1. / Санкт-Петербургский государственный горный институт имени Г. В. Плеханова, Санкт-Петербург, 1994. 235 с.: ил.

2. Дубинкин Д. М. Обоснование необходимости создания тяжелых платформ для открытых горных работ // Горное оборудование и электромеханика - 2020. - № 4 (150). - С. 59-64.

3. Дубинкин Д. М. Современное состояние техники и технологий в области автономного управления движением транспортных средств угольных карьеров // Горное оборудование и электромеханика - 2019. - № 6 (146). - С. 8-15.

4. Дубинкин Д. М., Садовец В. Ю., Котиев Г. О., Карташов А. Б. Исследование процесса транспортирования вскрышных пород и угля на разрезах // Техника и технология горного дела. - 2019. № 4 (7). - C. 50-66.

5. Paolo Tamburrano, Andrew R. Plummer, Elia Distaso, Riccardo Amirante, A Review of Direct Drive Proportional Electrohydraulic Spool Valves: Industrial State-of-the-Art and Research Advancements // Journal of Dynamic Systems Measurement and Control, August 2018, 17 p.

6. Jerry Boza, Design and Validation of an Electro-Hydraulic Pressure-Control Valve and Closed-Loop Controller // Western Michigan University, 12-2016, 128 p.

7. Jin-gang Liu, Gao-sheng Wang, Tian-heng Peng, and Sheng-qiang Jiang, Numerical Simulation of Solid Particle Erosion in Aluminum Alloy Spool Valve // Multiscale and Multiphase Computational Particle Technology, Volume 2019, $16 \mathrm{p}$.

8. Robert Jasionowski, Wojciech Polkowski, Dariusz Zasada, The Destruction Mechanism of Titanium Subjected to Cavitation Erosion // Key Engineering Materials, April 2016, 7 p.

9. Yuan-Jian Yang, Peng Weiwen, Debiao Meng, Shun-Peng Zhu, Reliability analysis of direct drive electrohydraulic servo valves based on a wear degradation process and individual differences // Proceedings of the Institution of Mechanical Engineers Part O Journal of Risk and Reliability, November 2014, 11 p.

10.Nuray Kayakol, Cavitation modelling in micro channels // Conference Paper, May 2016, 7 p.

11.Lomakin V. O, Kuleshova M. S., Kraeva E. A., Fluid flow in the throttle channel in the presence of cavitation // Dynamics and Vibroacoustics of Machines, Procedia Engineering 106 (2015 ) p. 27-35. 
12.Pavel Rudolf , Martin Juliš, Lenka Klakurková, Pavel Gejdoš and Martin Hudec, Cavitation erosion testing of different cavitation-resistent materials and coatings using the cavitating jet method // IOP Conf. Series: Earth and Environmental Science 240 (2019) 062057, 11 p.

13.Joost Rooze, Matthieu Andre', Gert-Jan S. van der Gulik, David Ferna'ndez-Rivas, Johannes G. E. [et al.], Hydrodynamic cavitation in micro channels with channel sizes of 100 and 750 micrometers // Microfluid Nanofluid (2012) 12, p. 499-508.

14.Araz Sheibani Aghdam, Morteza Ghorbani, Gokberk Deprem, Fevzi Çakmak Cebeci, Ali Koşar, A New Method for Intense Cavitation Bubble Generation on Layer-by-Layer Assembled SLIPS // Scientific Reports | (2019) 9:11600, 13 p.

15.Ji Pei, Majeed Koranteng Osman, Wenjie Wang, Desmond Appiah, Tingyun Yin, Qifan Deng, A Practical Method for Speeding up the Cavitation Prediction in an Industrial Double-Suction Centrifugal Pump // Energies 2019, 12, 2088, 20 p.

16.Shaimaa Saeed, Tarek M. Aboul-Fotouh, Ibrahim Ashour, A Current Viscosity of Different Egyptian Crude Oils: Measurements and Modeling Over a Certain Range of Temperature and Pressure // Saeed et al., J Pet Environ Biotechnol 2016, 7:6, 8 p.

17.Darko M Knežević, Darko Lovrec, Jocanović Mitar, Velibor Karanovic, Determination of Pressure Losses in Hydraulic Pipeline Systems by Considering Temperature and Pressure // Strojniski Vestnik, April 2009, 8 p.

18.Darko Knežević, Vladimir Savić, Mathematical modeling of changing of dynamic viscosity, as a function of temperature and pressure, of mineral oils for hydraulic systems // Mechanical Engineering Vol. 4, No 1, 2006, pp. 27-34.

19.Scott Bair, Paul Michael, Modelling the pressure and temperature dependence of viscosity and volume for hydraulic fluids // International Journal of Fluid Power 11 (2010) No. 2 pp. 37-42.

\section{REFERENCES}

1. A. A. Kuleshov, Design and operation of quarry vehicles. Handbook. Part 1. / St. Petersburg State Mining Institute named after G. V. Plekhanov, St. Petersburg, 1994. 235 p.: ill.

2. Dubinkin D. M. Justification of the need to create heavy platforms for open-pit mining. Mining Equipment and Electromechanics, 2020, no.4 (150), pp. 59-64.

3. Dubinkin D. M. Current state of engineering and technology in the field of autonomous traffic control of coal mine vehicles // Mining equipment and electromechanics No. 6, 2019, p. 8-15.

4. Dubinkin D. M., Sadovets V. Yu., Kotiev G. O., Kartashov A. V. (2019) Overburden and coal transportation research at open pit mines, Journal of mining and geotechnical engineering, 4(7):50

5. Paolo Tamburrano, Andrew R. Plummer, Elia Distaso, Riccardo Amirante, A Review of Direct Drive Proportional Electrohydraulic Spool Valves: Industrial State-of-the-Art and Research Advancements // Journal of Dynamic Systems Measurement and Control, August 2018, 17 p.

6. Jerry Boza, Design and Validation of an Electro-Hydraulic Pressure-Control Valve and Closed-Loop Controller // Western Michigan University, 12-2016, 128 p.

7. Jin-gang Liu, Gao-sheng Wang, Tian-heng Peng, and Sheng-qiang Jiang, Numerical Simulation of Solid Particle Erosion in Aluminum Alloy Spool Valve // Multiscale and Multiphase Computational Particle Technology, Volume 2019, 16 p.

8. Robert Jasionowski, Wojciech Polkowski, Dariusz Zasada, The Destruction Mechanism of Titanium Subjected to Cavitation Erosion // Key Engineering Materials, April 2016, 7 p.

9. Yuan-Jian Yang, Peng Weiwen, Debiao Meng, Shun-Peng Zhu, Reliability analysis of direct drive electrohydraulic servo valves based on a wear degradation process and individual differences // Proceedings of the Institution of Mechanical Engineers Part O Journal of Risk and Reliability, November 2014, 11 p.

10.Nuray Kayakol, Cavitation modelling in micro channels // Conference Paper, May 2016, 7 p.

11.Lomakin V. O, Kuleshova M. S., Kraeva E. A., Fluid flow in the throttle channel in the presence of cavitation // Dynamics and Vibroacoustics of Machines, Procedia Engineering 106 (2015 ) p. 27-35.

12.Pavel Rudolf, Martin Juliš, Lenka Klakurková, Pavel Gejdoš and Martin Hudec, Cavitation erosion testing of different cavitation-resistent materials and coatings using the cavitating jet method // IOP Conf. Series: Earth and Environmental Science 240 (2019) 062057, 11 p.

13.Joost Rooze, Matthieu Andre', Gert-Jan S. van der Gulik, David Ferna'ndez-Rivas, Johannes G. E. [et al.], Hydrodynamic cavitation in micro channels with channel sizes of 100 and 750 micrometers // Microfluid Nanofluid (2012) 12, p. 499-508.

14.Araz Sheibani Aghdam, Morteza Ghorbani, Gokberk Deprem, Fevzi Çakmak Cebeci, Ali Koşar, A New Method for Intense Cavitation Bubble Generation on Layer-by-Layer Assembled SLIPS // Scientific Reports | (2019) 9:11600, 13 p.

15.Ji Pei, Majeed Koranteng Osman, Wenjie Wang, Desmond Appiah, Tingyun Yin, Qifan Deng, A Practical 
Method for Speeding up the Cavitation Prediction in an Industrial Double-Suction Centrifugal Pump // Energies 2019, 12, 2088, 20 p.

16.Shaimaa Saeed, Tarek M. Aboul-Fotouh, Ibrahim Ashour, A Current Viscosity of Different Egyptian Crude Oils: Measurements and Modeling Over a Certain Range of Temperature and Pressure // Saeed et al., J Pet Environ Biotechnol 2016, 7:6, 8 p.

17.Darko M Knežević, Darko Lovrec, Jocanović Mitar, Velibor Karanovic, Determination of Pressure Losses in Hydraulic Pipeline Systems by Considering Temperature and Pressure // Strojniski Vestnik, April 2009,8 p.

18.Darko Knežević, Vladimir Savić, Mathematical modeling of changing of dynamic viscosity, as a function of temperature and pressure, of mineral oils for hydraulic systems // Mechanical Engineering Vol. 4, No 1, 2006, pp. 27-34.

19.Scott Bair, Paul Michael, Modelling the pressure and temperature dependence of viscosity and volume for hydraulic fluids // International Journal of Fluid Power 11 (2010) No. 2 pp. 37-42.

Поступило в редакцию 10.12.2020

Received 10 December 2020 